\title{
Visual Evoked Potential Characterization of Rabbit Animal Model For Retinal Prosthesis Research
}

\author{
Massoud L Khraiche ${ }^{1,2,4}$, Sharif El Emam ${ }^{3}$, Abraham Akinin ${ }^{1,4}$, Gert Cauwenberghs ${ }^{1,4}$, William Freeman ${ }^{3}$ and \\ Gabriel A. Silva ${ }^{1,2,3,4}$
}

\begin{abstract}
Visual evoked potentials (VEP) are used to confirm the function of prosthetic devices designed to stimulate retinas with damaged photoreceptors in vivo. In this work, we focus on methods and experimental consideration for recording visual evoked potential in rabbit models and assesses the use for retinal prosthesis research. We compare both invasive and noninvasive methods for recording VEPs, the response of the rabbit retina to various light wavelengths and intensities, focal vs. full field stimulation, and the effect of light bleaching on the retinal response.
\end{abstract}

\section{INTRODUCTION}

Degenerative diseases targeting the sensory photoreceptors in the retina lead to blindness. Currently, there are many ongoing efforts to interface with the retina for the purpose of stimulating the remaining neural layers to reproduce vision. In vivo testing of these devices to confirm function in an animal model typically follows basic in vitro tests that confirm stimulation of the retina. The types of animals used in retinal prosthesis research vary and different groups usually rely on rats, cats, dogs, sheep and minipigs as well as rabbits (see Bertschinger et al, for a detailed review). The rabbit animal model is often used in glaucoma and vitreoretinopathy research. There is extensive research using the rabbit animal model for retinal prosthesis testing in spite of the lack of a macula. This can be attributed to the advantages of using rabbit model including the size of the eye reducing surgical complications and the excellent wound healing.

VEPs (visual evoked potentials) are recorded in the visual cortex of animals in response to light stimulation via either needle electrodes placed in the scalp or skull burr holes. Light polarizes photoreceptor cells induces change in the gradient release of neural transmitters targeting the bipolar and horizontal cells. These cells in turn stimulate the ganglion cells in the inner retina that have axons extending via the optic tract and project to the lateral geniculate nucleus. The visual signal is relayed from LGN to the visual cortex through the optic radiation.

Studying retinal activation in response to stimulation in vitro consists of monitoring function of ganglion cells by pressing retinal explants directly onto Microelectrode arrays (MEA). The same concept is difficult to reproduce in vivo. Recording single unit activity from ganglion cells in live animals is challenging due to the anatomy of the eye, which restricts access to the ganglion cells (Single unit recording requires close proximity between the recording electrodes

1) Department of Bioengineering, University of California, San Diego, California,

2) Jacob's Retina Center

3) Department of Ophthalmology,

4) Institute of Engineering and Medicine

U.S. Government work not protected by U.S. copyright and ganglion cells). Our literature search found only one example of direct ganglion cell recording in an animal (Baig-Silva and Hetling (2005)). An alterative is recording a visual evoked potential from the visual cortex or, in case of direct electrical stimulation, an electrical evoked potentials (EEP). Another advantage of EEP recording is verifying that the signal evoked by retinal stimulation reaches the visual cortex.

In this work, we focus on methods of recording visual evoked potential in rabbit models and strategies to understand how it can be used for retinal prosthesis research. We compare both invasive and noninvasive methods for recording VEPs, the response of the rabbit retina to various light wavelengths and intensities, focal vs. full field stimulation, and the effect of light bleaching on the retinal response.

\section{MATERIALS AND METHODS}

\section{A. Animal surgery and electrode placement:}

Dutch belted pigmented rabbits $(2 \pm 3 \mathrm{~kg})$ used in the study were initially anesthetized using ketamine hydrochloride (50 $\mathrm{mg} / \mathrm{kg}$ ) and xylazine $(5 \mathrm{mg} / \mathrm{kg})$. The animal is placed in a stereotaxic frame. To prep the animal for surgery, scalp fur is shaved and the scalp is scrubbed with a gauze sponge starting at the center of the proposed incision and working in a circular pattern to the periphery. Animals are given Buprenorphine $0.3-0.5 \mathrm{mg} / \mathrm{kg}$ via a subcutaneous injection. Skin is incised, periosteum is opened, and the skull is exposed. Two skull burr holes are made, one at $11 \mathrm{~mm}$ behind the bregma and one at $4 \mathrm{~mm}$ from the sagittal suture overlying the visual cortex using a $1.5 \mathrm{~mm}$ diameter electric drill.

Two stainless-steel screws are placed in the burr holes (on

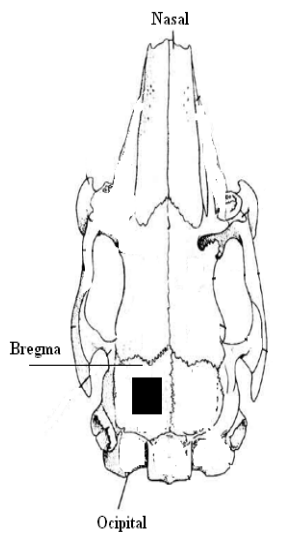

Fig.1: shows anatomical location of screw electrode. each side of the sagittal suture, see Fig. 1). A reference electrode is placed behind the ear. During the surgical procedure, the rabbit is monitored for normal breathing rates visually and heart rate with a pulse oximeter, and kept warm with heating blankets. Electrode location varies between studies (table 1).

B. VEP recording and Light stimulation

Visual evoked potentials are recorded using animal physiology UBA-4204 Universal 
Biomedical Amplifier (LKC, USA). Light flashes are set to $1 \mathrm{~Hz}$ frequency and $1,500,000$ candlepower at maximum stimulation and 93,000 candlepower at low stimulation with a flash duration of 10 microseconds. VEP waveforms are

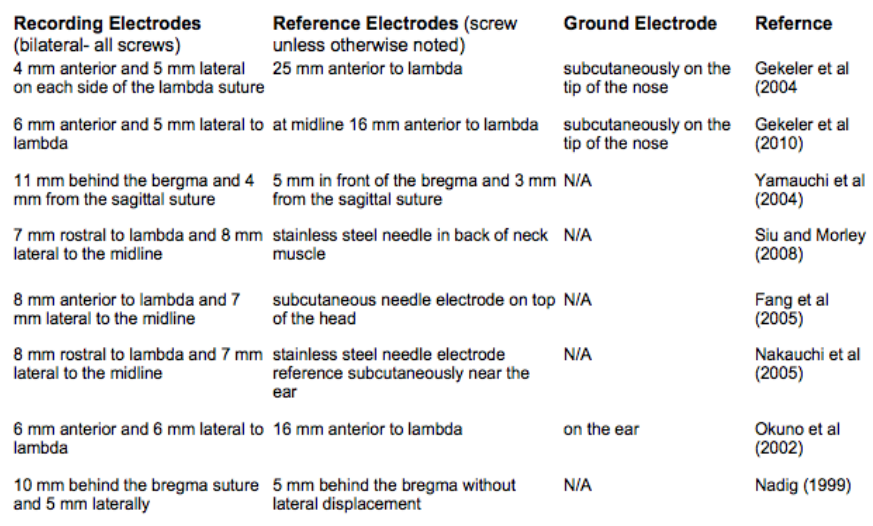

Table1: Shows varying electrode type and location reported in literature.

averaged over 80 pulses.

\section{Bleaching}

Fiber optics lights are used to bleach the retina of the anesthetized rabbit. Light intensity of 100K Lux was applied to both rabbit eyes for 15 minutes. VEP measurements took place 60 minutes after bleaching.

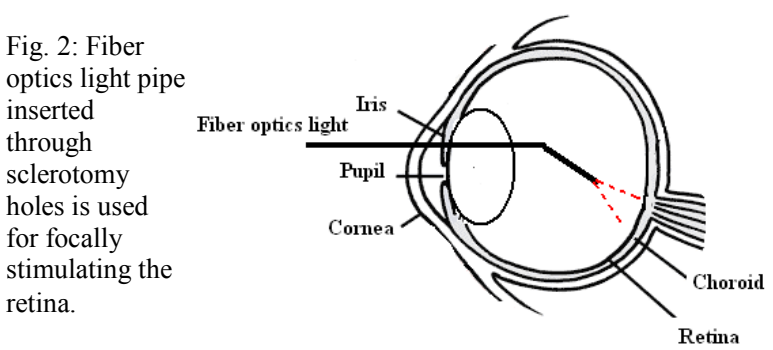

\section{Vitrectomy and focal light stimulation:}

Rabbits were anesthetized with a cocktail of $35 \mathrm{mg} / \mathrm{kg}$ ketamine and $5 \mathrm{mg} / \mathrm{kg}$ xylazine. A drop or 2 of proparacaine can be administered to numb the cornea.. Vitrectomies are performed under general anesthesia and take about 15-20 minutes per eye. The eye is proptosed by holding the superior and inferior rectus muscles using Nylon sutures. The eye is prepped with Betadine, irrigated with sterile BSS, and surgically draped. A drop of Goniosol is placed on the cornea, and a cover slip is set on top. Vitrectomies are performed using an Alcon Accurus machine. In the rabbit, sclerotomy sites are normally of a 20 -gauge size. In a full vitrectomy procedure, three sclerotomies are made--one for an infusion cannula connected to a BSS or Lactated Ringer's fluid bottle to maintains the eye pressure. The other 2 sclerotomies are for various surgical instruments including a light pipe, the vitrectomy probe, and vitrectomy scissors. The irrigating fluid replaces the vitreous as it is removed by the vitrector under the operating microscope, and using the fiber-optic light for illumination inside the eye. If the procedure lasts longer than 30 minutes, the animal's body heat will be maintained with warm water blankets.

\section{RESULTS}

\section{A. Comparison of Needle vs. Screw electrodes:}

Both screw and needle electrodes are used in literature to record VEPs. The needle electrode is placed in the scalp while the screw electrode is inserted through burr holes in the animal's skull. The shape of the VEP varies depending

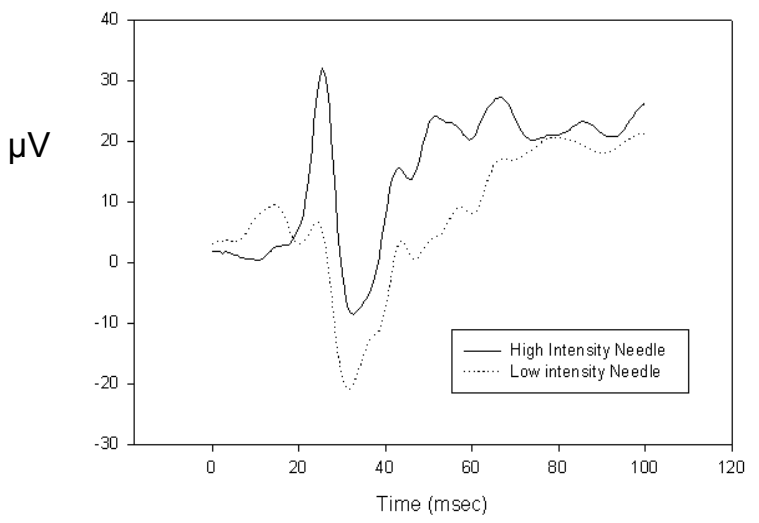

on

Fig. 3: VEP in response to high and low full field light stimuli with needle electrodes.

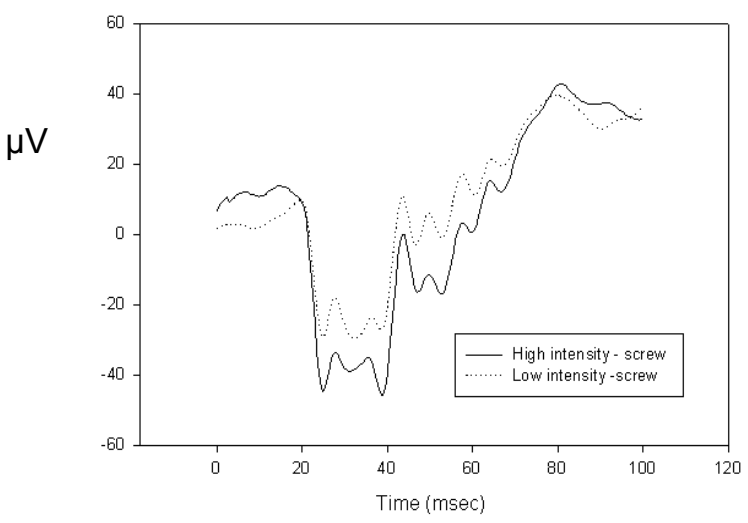

Fig. 4: VEP in response to high and low full field light stimuli with screw electrodes.

the electrode and the height of the VEP waveform is larger when using screw electrodes than it is using needle electrodes (Fig. 3 and Fig. 4) recorded from the same animal.

\section{B. Focal stimulation of the retina:}

Using a fiber optic light we were able to focally stimulate the retina to produce VEP waveforms with a minimal area of stimulation of $10 \mathrm{~mm}^{2}$. Fig. 5 shows VEP waveforms in response to high and low light intensity. We attempted smaller stimulation areas but it resulted in a nondetectable VEP waveforms. 


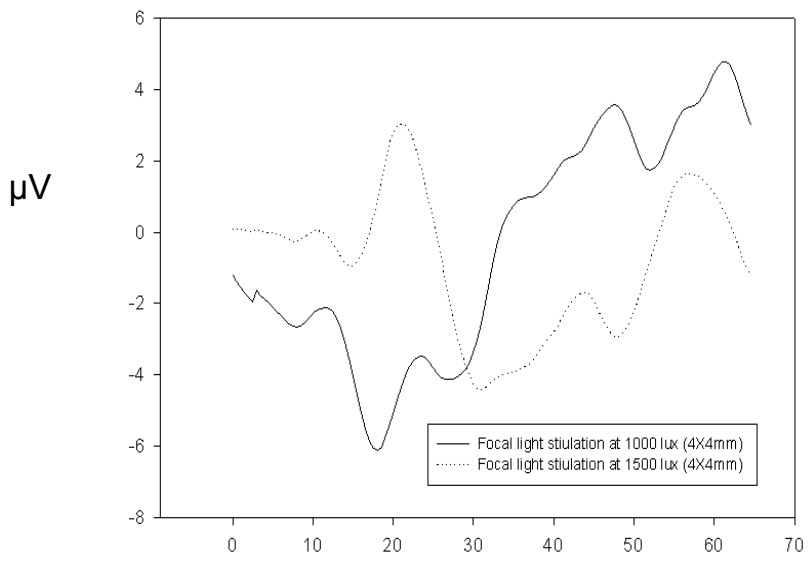

Fig. 5: Focal light stimulation of rabbit retina at two different light intensities.

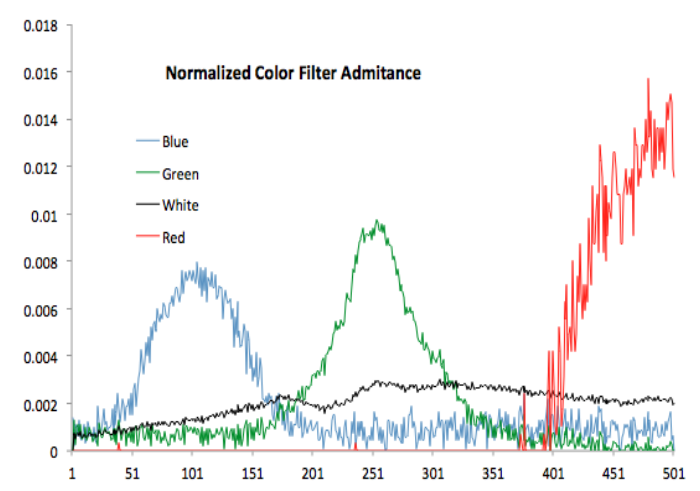

Fig. 6: Normalized color filter admittance used to stimulate the rabbit retina

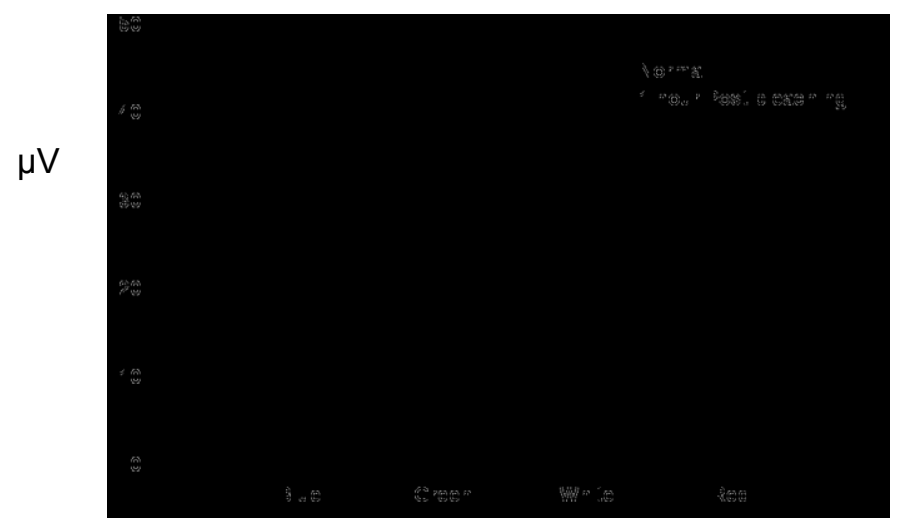

Fig. 7: Responsiveness of rabbit retina.

\section{Wavelength dependence of retinal response:}

We stimulated the rabbit retina with three different light filters (full field stimulation). The normalized admittance of the filters used for stimulation is shown in Fig.6. The responsivity of the rabbit retina varies across wavelengths and Fig. 7 shows the amplitude of collected VEP in response to full field stimulation via RGB filters and white light. The response to red light is very low.

\section{Effect of bleaching:}

Rabbit retina was exposed to high intensity light for the purposes of bleaching the photoreceptors and abolishing native retina response. Data in Fig. 7 shows reduction in the amplitude of VEP in response to retina stimulation 60 minutes post bleaching.

\section{DISCUSSION AND CONCLUSION}

\section{A. Screw vs. VEP:}

The experimenter has the choice of using screw electrodes versus using needle electrodes. Screw electrodes require invasive procedure for opening the scalp and burring holes in the animal's head. This introduces more complications when looking at survival surgeries, mainly due to large open wound in the animal's head. On the other hand, needle electrodes offer quick and noninvasive measurement and animals can be implanted chronically with a device and checked continuously. Our results show that needle electrodes have smaller VEP amplitudes when compared to VEP peaks obtained from screw electrodes. This can really come into play when activating small areas of the retina. The experimenter is faced with a trade off between the amplitude of the VEPs and the invasiveness of the procedure.

\section{B. The rabbit model for VEP and retinal prosthesis:}

As we mentioned, the rabbit model is a very useful model for retinal prosthesis research in spite of A) Lack of a macular and B) Lack of a degenerative model. The latter forces experimenters to find a way around abolishing or limiting native retinal response when using photovoltaic devices to stimulate the retina. The response of the natural retina can confound the results. Data in Fig. 7 shows native retina response to full field light stimulation characterized as the peak of VEP waveform. The wavelength of light can have a large effect on the response. This can come into play for stimulation using photovoltaic devices that have a different responsivity plot.

\section{Bleaching:}

Bleaching the photoreceptors with a high intensity light is not an uncommon approach to abolish or reduce the contribution from the native retina. Bleaching is basically the reduction in light sensitivity of photoreceptors in response to large photoconversion of rhodopsin. We attempted to investigate whether the photoreceptors response can be abolished to make way for retina stimulation via implanted prosthetic devices. As Fig. 7 showed, there is a reduction in the photoresponse to all colors (at 60 minutes post bleaching), but only completely abolished for red. In fact, from our data, red light stimulation appears to be the 
best light to be used to stimulate the retina while ensuring little contribution from the native retina photoreceptors.

\section{Focal light stimulation:}

Focal stimulation of the retina is aimed at investigating the affect of stimulating small areas of the retina on the VEP waveform.Focal stimulation via electric stimulation is well characterized by Nadig et al, were EEPs are compared against the area of stimulation. The author shows the effect of reducing the size of the stimulation site on the amplitude of the EEP waveform. In this work, we attempted to focally stimulate the retina to study changes in the VEP waveform. Although we detected changes in the waveform for high and low white light intensities when stimulating the retina with a 2X2mm light stimulus, we were not able to see distinguishable peaks in the VEP waveforms (data not shown). Larger areas of stimulation at higher intensities show clearer VEP peaks, Fig. 5.

\section{E. Rabbit Retina Responsivity:}

In this work, we characterized the response of a rabbit retina using three different colored filters and white light. Our group is building a photovoltaic device to stimulate the retina and replace the function of the photoreceptors. The response of the native retina in the rabbit animal model can confound the response to any prosthetic device using a photovoltaic component. This can be avoided by using animal models of retinal degeneration with a reduced response to light that are not available in rabbits. Understanding the response of the retina and the potential for abolishing or reducing it can help design experiments to confirm retinal stimulation in rabbits using photovoltaic stimulation.

\section{CONCLUSION}

Data from VEP is useful and well documented for use in investigating retinal activation. In this work, we highlight strategies and experimental methodologies for using VEP to investigate retinal activation via prosthetic devices with emphasis on photovoltaic devices. Our data shows red light can be used for stimulation with little contribution from the native retina. Focal light stimulation is possible but there are limitation based on the smallest area that can be used to stimulate the retina.

\section{REFERENCES}

[1] X. Fang, H. Sakaguchi, T. Fujikado, M. Osanai, H. Kanda, Y. Ikuno, M. Kamei, M. Ohji, D. Gan, J. Choi, T. Yagi and Y. Tano, Graef Arch Clin Exp, 2005, 243, 49-56.

[2] F. Gekeler, K. Kobuch, G. Blatsios, E. Zrenner and K. Shinoda, Jpn J Ophthalmol, 2010, 54, 467-475.

[3] F. Gekeler, K. Kobuch, H. N. Schwahn, A. Stett, K. Shinoda and E. Zrenner, Graef Arch Clin Exp, 2004, 242, 587-596.

[4] F. Gekeler, A. Kopp, H. Sachs, D. Besch, U. Greppmaier, E. Zrenner, K. U. Bartz-Schmidt and P. Szurman, Brit J Ophthalmol, 2010, 94, 843-847.

[5] M. N. Nadig, Clin Neurophysiol, 1999, 110, 1545-1553

[6] K. Nakauchi, T. Fujikado, H. Kanda, T. Morimoto, J. S. Choi, Y. Ikuno, H. Sakaguchi, M. Kamei, M. Ohji, T. Yagi, S. Nishimura, H. Sawai, Y. Fukuda and Y. Tano, Graef Arch Clin Exp, 2005, 243, 169174.
[7] M. V. Narayanan, J. F. Rizzo, D. Edell and J. L. Wyatt, Invest Ophth Vis Sci, 1994, 35, 1380-1380.

[8] T. Okuno, H. Oku, T. Sugiyama, Y. Yang and T. Ikeda, Invest Ophth Vis Sci, 2002, 43, 784-789.

[9] H. Sailer, K. Shinoda, G. Blatsios, K. Kohler, L. Bondzio, E. Zrenner and F. Gekeler, Graef Arch Clin Exp, 2007, 245, 1169-1178.

[10] T. L. Siu and J. W. Morley, Eur J Neurosci, 2008, 28, 484-490.

[11] G. Willmann, K. Schaferhoff, M. D. Fischer, B. Arango-Gonzalez, S. Bolz, L. Naycheva, T. Rock, M. Bonin, K. U. Bartz-Schmidt, E. Zrenner, A. Schatz and F. Gekeler, Invest Ophth Vis Sci, 2011, 52, 7529-7537.

[12] D. R. Bertschinger, E. Beknazar, M. Simonutti, A. B. Safran, J. A. Sahel, S. G. Rosolen, S. Picaud and J. Salzmann, Graef Arch Clin Exp, 2008, 246, 1505-1517. 